\title{
Robust Exponential Synchronization for a Class of Master-Slave Distributed Parameter Systems with Spatially Variable Coefficients and Nonlinear Perturbation
}

\author{
Chengdong Yang, ${ }^{1,2}$ Jianlong Qiu, ${ }^{3,4}$ Kejia Yi, ${ }^{5}$ Xiangyong Chen, ${ }^{3}$ Ancai Zhang, ${ }^{6}$ \\ Xiao Chen, ${ }^{1,2}$ and Liuqing Yang ${ }^{7}$ \\ ${ }^{1}$ School of Informatics, Linyi University, Linyi 276005, China \\ ${ }^{2}$ Provincial Key Laboratory for Network Based Intelligent Computing, Jinan 250022, China \\ ${ }^{3}$ School of Science, Linyi University, Linyi 276005, China \\ ${ }^{4}$ Department of Electrical and Computer Engineering, University of Rhode Island, Kingston, RI 02881, USA \\ ${ }^{5}$ Science and Technology on Underwater Acoustic Antagonizing Laboratory, Systems Engineering Research Institute of CSSC, \\ Beijing 100036, China \\ ${ }^{6}$ School of Automobile Engineering, Linyi University, Linyi 276005, China \\ ${ }^{7}$ School of Electronic and Information Engineering, Xian Jiaotong University, Xian 710049, China
}

Correspondence should be addressed to Jianlong Qiu; qiujianlong@lyu.edu.cn

Received 16 October 2014; Revised 12 April 2015; Accepted 15 April 2015

Academic Editor: Anna Vila

Copyright (C) 2015 Chengdong Yang et al. This is an open access article distributed under the Creative Commons Attribution License, which permits unrestricted use, distribution, and reproduction in any medium, provided the original work is properly cited.

\begin{abstract}
This paper addresses the exponential synchronization problem of a class of master-slave distributed parameter systems (DPSs) with spatially variable coefficients and spatiotemporally variable nonlinear perturbation, modeled by a couple of semilinear parabolic partial differential equations (PDEs). With a locally Lipschitz constraint, the perturbation is a continuous function of time, space, and system state. Firstly, a sufficient condition for the robust exponential synchronization of the unforced semilinear master-slave PDE systems is investigated for all admissible nonlinear perturbations. Secondly, a robust distributed proportional-spatial derivative (P-sD) state feedback controller is desired such that the closed-loop master-slave PDE systems achieve exponential synchronization. Using Lyapunov's direct method and the technique of integration by parts, the main results of this paper are presented in terms of spatial differential linear matrix inequalities (SDLMIs). Finally, two numerical examples are provided to show the effectiveness of the proposed methods applied to the robust exponential synchronization problem of master-slave PDE systems with nonlinear perturbation.
\end{abstract}

\section{Introduction}

The study on master-slave systems has become more important for theoretical and practical points in many fields, including communication, mechanical systems, robotics, chemical reactions, and biological systems [1-9]. Ever since the discovery of Christian Huygens in 1665 on the synchronization of two pendulum clocks [10], synchronization has received considerable attention for a long time as a typical collective behavior and a basic motion in nature with potential applications in many different areas including secure communication, chaos generators design, chemical reactions, biological systems, and information science [1126]. The theory of synchronization for master-slave systems, which aims to control the slave system so that the output of the slave system follows the output of the master system [27], is a recent research area extensively investigated nowadays in many industrial and technical processes, such as unmanned aerial vehicle (UAV) team, vehicular platoons, rendezvous of space shuttles, and many other practical control systems (see, e.g., $[28,29]$ and the references therein).

The existing works most considered dynamical behavior of master-slave systems described by ordinary differential equations (ODEs) or delay differential equations (DDEs), and 
a variety of synchronization criteria have been presented [1416]. In practice, however, the outputs, inputs, and process states with relevant parameters usually vary both temporally and spatially in nature, and thereby their behavior depending on time and spatial position could be described by distributed parameter systems (DPSs) modeled by partial differential equations (PDEs). Unfortunately, few works have investigated the synchronization of master-slave PDE systems.

As a result of the infinite-dimensional nature of masterslave PDE systems, the existing finite-dimensional control theory and techniques for the master-slave ODE systems are difficult to be directly employed for the control design of master-slave PDE systems. In this situation, it is important to study the synchronization problem of master-slave PDE systems. Therefore, some researchers have paid attention to the study of synchronization of master-slave PDE systems [17-29], where "design-then-reduce" approach was employed to take the full advantage of the original PDE model for the controller design [30-34]. References [35-37] researched synchronization of neural networks with reaction-diffusion terms. Yuan et al. proposed synchronization of the coupled distributed parameter system with time delay via P-sD control [38]. Yang et al. proposed exponential synchronization for complex spatiotemporal networks with space-varying coefficients via P-sD control [39]. Wang et al. studied exponential synchronization for networked linear PDE systems via boundary control [40]. However, to the best of our knowledge, few results are available on the exponential synchronization of master-slave PDE systems with nonlinear perturbation and spatially variable coefficients, which motives the present investigation.

In this paper, we will deal with the problem of robust exponential synchronization for master-slave parabolic PDE systems with spatially variable coefficients and spatiotemporally variable nonlinear perturbation. Initially, a masterslave parabolic PDE model is discussed, and then a synchronization error dynamic of PDE systems is developed in spatial domain. Then, on the basis of Lyapunov's direct method and the technique of integration by parts, a spatial differential linear matrix inequality (SDLMI) based condition for the robust exponential synchronization of the unforced semilinear master-slave PDE systems is studied. Once the unforced semilinear master-slave PDE systems cannot achieve robust exponential synchronization, distributed proportional-spatial derivative (P-sD) control design is developed to achieve that the closed-loop slave system which is exponentially synchronized with the master system with a given decay rate for all admissible nonlinear perturbations in terms of SDLMI. Furthermore, the SDLMI optimization problem can be approximately solved by the finite difference method and LMI optimization techniques $[41,42]$. Finally, the simulation study on the exponentially synchronous control of a master-slave PDE systems with nonlinear perturbation is given to show the effectiveness of the proposed design method.

The remainder of this paper is organized as follows. The problem formulation and preliminaries are given in Section 2. Section 3 provides the exponential synchronization analysis of the unforced master-slave PDE systems.
Robust P-sD controller is designed in Section 4. Section 5 presents two examples on master-slave PDE systems to illustrate the effectiveness of the proposed method. Finally, Section 6 offers some concluding remarks.

Notations. The following notations will be employed throughout this paper. $\mathfrak{R}, \mathfrak{R}^{n}$, and $\mathfrak{R}^{m \times n}$ denote the set of all real numbers, $n$-dimensional Euclidean space, and the set of all $m \times n$ matrices, respectively. $\|\cdot\|$ and $\langle\cdot, \cdot\rangle_{\mathfrak{R}^{n}}$ denote the Euclidean norm and inner product for vectors, respectively. Identity matrix, of appropriate dimensions, will be denoted by $\mathbf{I}$. For a symmetric matrix $\mathbf{X}, \mathbf{X}>0(\mathbf{X}<0, \mathbf{X} \leq 0$, resp.) means that $\mathbf{X}$ is positive definite (negative definite, negative semidefinite, resp.). The space-varying symmetric matrix function $\mathbf{P}(x), x \in\left[l_{1}, l_{2}\right]$, is positive definite (negative definite, negative semidefinite, resp.), if $\mathbf{P}(x)>0(\mathbf{P}(x)<$ $0, \mathbf{P}(x) \leq 0$, resp.) for each $x \in\left[l_{1}, l_{2}\right] . \lambda(\cdot)$ represents the eigenvalue of a matrix. The superscript " $T$ " is used for the transpose of a vector or a matrix. $\mathscr{W}^{l, 2}\left(\left[l_{1}, l_{2}\right] ; \mathfrak{R}^{n}\right)$ is a Sobolev space of absolutely continuous $n$-dimensional vector functions $\boldsymbol{\omega}(x):\left[l_{1}, l_{2}\right] \rightarrow \mathfrak{R}^{n}$ with square integrable derivatives $d^{k} \boldsymbol{\omega}(x) / d x^{k}$ of the order $k \geq 1$ and with the norm $\|\boldsymbol{\omega}(\cdot)\|_{\mathscr{W}^{l, 2}}^{2}=\int_{l_{1}}^{l_{2}} \sum_{i=0}^{k}\left(d^{i} \boldsymbol{\omega}(x) / d x^{i}\right)^{T}\left(d^{i} \boldsymbol{\omega}(x) / d x^{i}\right) d x$. The symbol “*” is used as an ellipsis in matrix expressions that are induced by symmetry; for example,

$$
\begin{aligned}
& {\left[\begin{array}{cc}
\mathbf{P}+[\mathbf{M}+\mathbf{N}+*] & \mathbf{X} \\
* & \mathbf{Y}
\end{array}\right]} \\
& \triangleq\left[\begin{array}{cc}
\mathbf{P}+\left[\mathbf{M}+\mathbf{N}+\mathbf{M}^{T}+\mathbf{N}^{T}\right] & \mathbf{X} \\
\mathbf{X}^{T} & \mathbf{Y}
\end{array}\right] .
\end{aligned}
$$

\section{Problem Formulation}

Consider the following synchronization scheme of semilinear master-slave PDE systems with spatially variable coefficients and spatiotemporally variables as follows.

The master system $\mathscr{M}$ is as follows:

$$
\begin{aligned}
\mathbf{y}_{t}(x, t)= & \left(\boldsymbol{\Theta}_{1}(x) \mathbf{y}_{x}(x, t)\right)_{x}+\boldsymbol{\Theta}_{2}(x) \mathbf{y}_{x}(x, t) \\
& +\mathbf{A}(x) \mathbf{y}(x, t)+\mathbf{f}(\mathbf{y}(x, t), x, t), \\
\left.\mathbf{y}_{x}(x, t)\right|_{x=l_{1}}= & \left.\mathbf{y}_{x}(x, t)\right|_{x=l_{2}}=0, \\
\mathbf{y}(x, 0)= & \mathbf{y}_{0}(x),
\end{aligned}
$$

and the slave system $\mathcal{S}$ is as follows:

$$
\begin{aligned}
\mathbf{z}_{t}(x, t)= & \left(\boldsymbol{\Theta}_{1}(x) \mathbf{z}_{x}(x, t)\right)_{x}+\boldsymbol{\Theta}_{2}(x) \mathbf{z}_{x}(x, t) \\
& +\mathbf{A}(x) \mathbf{z}(x, t)+\mathbf{f}(\mathbf{z}(x, t), x, t) \\
& +\mathbf{G}(x) \mathbf{u}(x, t), \\
\left.\mathbf{z}_{x}(x, t)\right|_{x=l_{1}}= & \left.\mathbf{z}_{x}(x, t)\right|_{x=l_{2}}=0, \\
\mathbf{z}(x, 0)= & \mathbf{z}_{0}(x),
\end{aligned}
$$

where $\mathbf{y}(x, t)=\left[y_{1}(x, t), \ldots, y_{n}(x, t)\right]^{T}, \mathbf{z}(x, t)=\left[z_{1}(x, t), \ldots\right.$, $\left.z_{n}(x, t)\right]^{T} \in \mathfrak{R}^{n}$ are the state vectors, the subscripts $x$ and $t$ 
stand for the partial derivatives with respect to $x, t$, respectively, $x \in\left[l_{1}, l_{2}\right] \subset \Re$ and $t \in[0, \infty)$ are the spatial position and time, respectively, and $\mathbf{u}(x, t) \in \mathfrak{R}^{m}$ is the distributed control input. $\boldsymbol{\Theta}_{1}(x), \Theta_{2}(x)$, and $\mathbf{A}(x) \in \mathfrak{R}^{n \times n}$, $x \in\left[l_{1}, l_{2}\right]$, are real known matrix functions. $\mathrm{G}(x) \in \mathfrak{R}^{n \times m}$, $x \in\left[l_{1}, l_{2}\right]$, is a real known matrix function. $\mathbf{f}(\mathbf{y}(x, t), x, t)$ is $n$-dimensional real vector-valued spatiotemporally variable nonlinear perturbation with $\mathbf{f}(0, x, t)=0$ for all $x \in\left[l_{1}, l_{2}\right]$ and $t \geq 0$. Moreover, assume that $\mathbf{f}(\mathbf{y}(x, t), x, t)$ satisfies the following Lipschitz condition as follows.

Assumption 1. $\mathbf{f}(\mathbf{y}(x, t), x, t)$ is continuous with respect to its arguments and is locally Lipschitz continuous in $\mathbf{y}(x, t)$ on $\Omega$; that is, there exists a scalar $\chi>0$ such that the following inequality holds for all $\mathbf{y}(x, t), \widetilde{\mathbf{y}}(x, t) \in \Omega, t \geq 0$ and $x \in$ $\left[l_{1}, l_{2}\right]$ :

$$
\begin{aligned}
& \|\mathbf{f}(\mathbf{y}(x, t), x, t)-\mathbf{f}(\widetilde{\mathbf{y}}(x, t), x, t)\|_{2}^{2} \\
& \quad \leq x\|(\mathbf{y}(x, t)-\widetilde{\mathbf{y}}(x, t))\|_{2}^{2},
\end{aligned}
$$

where $\Omega \triangleq\left\{\mathbf{y}(x, t) \mid \sigma_{1, i}(x) \leq y_{i}(x, t) \leq \sigma_{2, i}(x), x \in\left[l_{1}, l_{2}\right]\right.$, $t \geq 0, i=1,2, \ldots, n\}$. Therefore, we have that

$$
\|\mathbf{f}(\mathbf{y}(x, t), x, t)\|_{2}^{2} \leq \chi\|\mathbf{y}(x, t)\|_{2}^{2} .
$$

Define the synchronization error $\mathbf{e}(x, t) \triangleq \mathbf{z}(x, t)-\mathbf{y}(x, t)$, and we have the following synchronization error system $\mathscr{E}$ :

$$
\begin{aligned}
\mathbf{e}_{t}(x, t)= & \left(\boldsymbol{\Theta}_{1}(x) \mathbf{e}_{x}(x, t)\right)_{x}+\boldsymbol{\Theta}_{2}(x) \mathbf{e}_{x}(x, t) \\
& +\mathbf{A}(x) \mathbf{e}(x, t)+\mathbf{F}(\mathbf{e}(x, t)) \\
& +\mathbf{G}(x) \mathbf{u}(x, t), \\
\left.\mathbf{e}_{x}(x, t)\right|_{x=l_{1}}= & \left.\mathbf{e}_{x}(x, t)\right|_{x=l_{2}}=0, \\
\mathbf{e}(x, 0)= & \mathbf{e}_{0}(x) \triangleq \mathbf{z}_{0}(x)-\mathbf{y}_{0}(x),
\end{aligned}
$$

where $\mathbf{F}(\mathbf{e}(x, t))=\mathbf{f}(z(x, t), x, t)-\mathbf{f}(\mathbf{y}(x, t), x, t)$.

Choose $\mathscr{H}^{n} \triangleq \mathscr{L}_{2}\left(\left[l_{1}, l_{2}\right] ; \mathfrak{R}^{n}\right)$ as the state space and the trajectory segment $\mathbf{e}(\cdot, t)=\left\{\mathbf{e}(x, t), x \in\left[l_{1}, l_{2}\right]\right\}$ as the state. Define the spatial differential operator $\mathscr{A}$ in $\mathscr{H}^{n}$ as

$$
\mathscr{A} \overline{\mathbf{e}}(t) \triangleq \frac{d}{d x}\left[\boldsymbol{\Theta}_{1}(x) \frac{d \overline{\mathbf{e}}(t)}{d x}\right]+\boldsymbol{\Theta}_{2}(x) \frac{d \overline{\mathbf{e}}(t)}{d x}
$$

with its domain

$$
\begin{aligned}
\mathscr{D} & (\mathscr{A}) \\
& =\left\{\overline{\mathbf{e}} \in \mathscr{W}^{2,2}\left(\left[l_{1}, l_{2}\right], \mathfrak{R}^{n}\right):\left.\overline{\mathbf{e}}_{x}\right|_{x=l_{1}}=0,\left.\overline{\mathbf{e}}_{x}\right|_{x=l_{2}}=0\right\} .
\end{aligned}
$$

Let $\mathcal{U} \triangleq \mathscr{L}_{2}\left(\left[l_{1}, l_{2}\right] ; \mathfrak{R}^{m}\right)$ be control input space and the trajectory segment $\mathbf{u}(\cdot, t)=\left\{\mathbf{u}(x, t), x \in\left[l_{1}, l_{2}\right]\right\}$ as the control input. Then the state-space description of the synchronization error system (6) can be rewritten as the following nonlinear abstract differential equation on the Hilbert space $\mathscr{H}^{n}[43]$ :

$$
\begin{aligned}
& \dot{\mathbf{e}}(t)=\mathscr{A} \mathbf{e}(t)+\widetilde{\mathbf{A}} \mathbf{e}(t)+\widetilde{\mathbf{F}}(\mathbf{e}(t))+\widetilde{\mathbf{G}} \mathbf{u}(t), \\
& \mathscr{D}(\mathscr{A}) \\
& \quad=\left\{\mathbf{e} \in \mathscr{W}^{2,2}\left(\left[l_{1}, l_{2}\right], \mathfrak{R}^{n}\right):\left.\mathbf{e}_{x}\right|_{x=l_{1}}=0,\left.\mathbf{e}_{x}\right|_{x=l_{2}}=0\right\}, \\
& \mathbf{e}(0)=\mathbf{e},
\end{aligned}
$$

where $\widetilde{\mathbf{A}} \triangleq \mathbf{A}(\cdot), \widetilde{\mathbf{G}} \triangleq \mathbf{G}(\cdot), \widetilde{\mathbf{F}}(\mathbf{e}(t)) \triangleq \mathbf{F}(\mathbf{e}(\cdot, t))$, and $\mathbf{e} \triangleq \mathbf{e}(\cdot) \epsilon$ $\mathscr{H}^{n}$.

Using (8), we get from (6) that

$$
\begin{aligned}
\mathbf{e}_{t}(x, t)= & \mathscr{A} \mathbf{e}(x, t)+\mathbf{A}(x) \mathbf{e}(x, t)+\mathbf{F}(\mathbf{e}(x, t)) \\
& +\mathbf{G}(x) \mathbf{u}(x, t) \\
\left.\mathbf{e}_{x}(x, t)\right|_{x=l_{1}}= & \left.\mathbf{e}_{x}(x, t)\right|_{x=l_{2}}=0 \\
\mathbf{e}(x, 0)= & \mathbf{e}_{0}(x)
\end{aligned}
$$

where $\mathbf{e}_{0}(x) \triangleq \mathbf{z}_{0}(x)-\mathbf{y}_{0}(x)$.

We introduce the following definition of the exponential synchronization for the master-slave systems (2) and (3) in the sense of norm $\|\cdot\|_{2}$.

Definition 2. For a given constant $\rho>0$, the master-slave systems (2)-(3) achieve $\rho$-exponential synchronization or exponential synchronization with a given decay rate $\rho$, if there exists a constant $\sigma>0$ such that the following inequality holds for any initial condition $\mathbf{e}_{0}(x), x \in\left[l_{1}, l_{2}\right]$ :

$$
\|\mathbf{e}(\cdot, t)\|_{2}^{2} \leq \sigma \exp (-2 \rho t)\left\|\mathbf{e}_{0}(\cdot)\right\|_{2}^{2}, \quad \forall t \geq 0 .
$$

It is easily seen from Definition 2 that the master-slave systems (2)-(3) achieve exponential synchronization with a given decay rate $\rho$ if and only if the error system (6) is exponentially stable with a given decay rate $\rho$.

For simplicity, when $\mathbf{u}(x, t) \equiv 0$, the master-slave systems (2)-(3) are referred to as unforced master-slave systems.

Lemma 3. For any two square integrable vector functions $\mathbf{a}(x)$, $\mathbf{b}(x) \in \mathfrak{R}^{n}, x \in\left[l_{1}, l_{2}\right] \subset \mathfrak{R}$, the following inequality holds for any positive scalar function $\alpha(x) \in \mathfrak{R}$ defined on interval $\left[l_{1}, l_{2}\right]$ :

$$
2\langle\mathbf{a}, \mathbf{b}\rangle \leq\langle\mathbf{a}, \alpha \mathbf{a}\rangle+\left\langle\mathbf{b}, \alpha^{-1} \mathbf{b}\right\rangle .
$$

Proof. It is easily found that the inequality $[\alpha(x) \mathbf{a}(x)-$ $\mathbf{b}(x)]^{T}[\alpha(x) \mathbf{a}(x)-\mathbf{b}(x)] \geq 0$ holds for any $x \in\left[l_{1}, l_{2}\right]$. Hence,

$$
\begin{aligned}
0 \leq & {[\alpha(x) \mathbf{a}(x)-\mathbf{b}(x)]^{T}[\alpha(x) \mathbf{a}(x)-\mathbf{b}(x)] } \\
= & \alpha^{2}(x) \mathbf{a}^{T}(x) \mathbf{a}(x)-2 \alpha(x) \mathbf{a}^{T}(x) \mathbf{b}(x) \\
& +\mathbf{b}^{T}(x) \mathbf{b}(x),
\end{aligned}
$$

which implies

$$
\begin{aligned}
2 \mathbf{a}^{T}(x) \mathbf{b}(x) \leq & \alpha(x) \mathbf{a}^{T}(x) \mathbf{a}(x) \\
& +\alpha^{-1}(x) \mathbf{b}^{T}(x) \mathbf{b}(x), \quad x \in\left[l_{1}, l_{2}\right] .
\end{aligned}
$$


Integrating inequality (14) from $l_{1}$ to $l_{2}$, we can obtain that

$$
\begin{aligned}
2 \int_{l_{1}}^{l_{2}} \mathbf{a}^{T}(x) \mathbf{b}(x) d x \leq & \int_{l_{1}}^{l_{2}} \alpha(x) \mathbf{a}^{T}(x) \mathbf{a}(x) d x \\
& +\int_{l_{1}}^{l_{2}} \alpha^{-1}(x) \mathbf{b}^{T}(x) \mathbf{b}(x) d x
\end{aligned}
$$

which means that inequality (12) holds. The proof is complete.

\section{Exponential Synchronization Analysis}

This section aims to analyze exponential synchronization for the unforced semilinear master-slave PDE systems (2)-(3).

We consider the following Lyapunov functional for the unforced synchronization error system (10):

$$
V(t)=\int_{l_{1}}^{l_{2}} \mathbf{e}^{T}(x, t) \mathbf{P}(x) \mathbf{e}(x, t) d x,
$$

where $\mathbf{P}(x)>0$ is a real $n \times n$ matrix function to be determined. The time derivative of $V(t)$ along the solution of system (10) is given by

$$
\begin{aligned}
\dot{V}(t) & +2 \rho V(t) \\
= & 2 \int_{l_{1}}^{l_{2}} \mathbf{e}^{T}(x, t) \mathbf{P}(x) \mathbf{e}_{t}(x, t) d x \\
& +2 \rho \int_{l_{1}}^{l_{2}} \mathbf{e}^{T}(x, t) \mathbf{P}(x) \mathbf{e}(x, t) d x \\
= & 2 \int_{l_{1}}^{l_{2}} \mathbf{e}^{T}(x, t) \mathbf{P}(x) \mathscr{A} \mathbf{e}(x, t) d x \\
& +2 \int_{l_{1}}^{l_{2}} \mathbf{e}^{T}(x, t) \mathbf{P A}(x) \mathbf{e}(x, t) d x \\
& +2 \int_{l_{1}}^{l_{2}} \mathbf{e}^{T}(x, t) \mathbf{P}(x) \mathbf{F}(\mathbf{e}(x, t)) \mathbf{e}(x, t) d x \\
& +2 \rho \int_{l_{1}}^{l_{2}} \mathbf{e}^{T}(x, t) \mathbf{P}(x) \mathbf{e}(x, t) d x .
\end{aligned}
$$

By integrating by parts and taking into account the boundary condition in (10), we have

$$
\begin{aligned}
& \int_{l_{1}}^{l_{2}} \mathbf{e}^{T}(x, t) \mathbf{P}(x)\left(\boldsymbol{\Theta}_{1}(x) \mathbf{e}_{x}(x, t)\right)_{x} d x \\
&=\left.\mathbf{e}^{T}(x, t) \mathbf{P}(x) \boldsymbol{\Theta}_{1}(x) \mathbf{e}_{x}(x, t)\right|_{x=l_{1}} ^{x=l_{2}} \\
&-\int_{l_{1}}^{l_{2}}\left(\mathbf{e}^{T}(x, t) \mathbf{P}(x)\right)_{x} \boldsymbol{\Theta}_{1}(x) \mathbf{e}_{x}(x, t) d x \\
&=-\int_{l_{1}}^{l_{2}} \mathbf{e}_{x}^{T}(x, t) \mathbf{P}(x) \Theta_{1}(x) \mathbf{e}_{x}(x, t) d x \\
&-\int_{l_{1}}^{l_{2}} \mathbf{e}^{T}(x, t) \mathbf{P}_{x}(x) \boldsymbol{\Theta}_{1}(x) \mathbf{e}_{x}(x, t) d x .
\end{aligned}
$$

According to the definition of $\mathscr{A}$ in (7) and considering (18), we have

$$
\begin{aligned}
\int_{l_{1}}^{l_{2}} \mathbf{e}^{T}(x, t) \mathbf{P}(x) \mathscr{A} \mathbf{e}(x, t) d x \\
=-\int_{l_{1}}^{l_{2}} \mathbf{e}_{x}^{T}(x, t) \mathbf{P}(x) \Theta_{1}(x) \mathbf{e}_{x}(x, t) d x \\
\quad-\int_{l_{1}}^{l_{2}} \mathbf{e}^{T}(x, t) \mathbf{P}_{x}(x) \Theta_{1}(x) \mathbf{e}_{x}(x, t) d x \\
\quad+\int_{l_{1}}^{l_{2}} \mathbf{e}^{T}(x, t) \mathbf{P}(x) \Theta_{2}(x) \mathbf{e}_{x}(x, t) d x .
\end{aligned}
$$

By Assumption 1 and Lemma 3, for any positive scalar function $\alpha(x) \in \Re, x \in\left[l_{1}, l_{2}\right]$, we have

$$
\begin{gathered}
2 \int_{l_{1}}^{l_{2}} \mathbf{e}^{T}(x, t) \mathbf{P}(x) \mathbf{F}(\mathbf{e}(x, t)) d x \leq \int_{l_{1}}^{l_{2}} \alpha^{-1}(x) \\
\cdot \mathbf{e}^{T}(x, t) \mathbf{P}(x) \mathbf{P}(x) \mathbf{e}(x, t) d x+\int_{l_{1}}^{l_{2}} \alpha(x) \\
\cdot \mathbf{F}^{T}(\mathbf{e}(x, t), \mathbf{y}(x, t)) \mathbf{F}(\mathbf{e}(x, t)) d x \leq \int_{l_{1}}^{l_{2}} \alpha(x) \\
\cdot \mathbf{e}^{T}(x, t) \mathbf{P}(x) \mathbf{P}(x) \mathbf{e}(x, t) d x+\chi \int_{l_{1}}^{l_{2}} \alpha^{-1}(x) \\
\cdot \mathbf{e}^{T}(x, t) \mathbf{e}(x, t) d x=\int_{l_{1}}^{l_{2}} \mathbf{e}^{T}(x, t) \\
\cdot\left[\alpha^{-1}(x) \mathbf{P}(x) \mathbf{P}(x)+\chi \alpha(x) \mathbf{I}\right] \mathbf{e}(x, t) d x .
\end{gathered}
$$

Substituting (19), (20) into (17), we obtain

$$
\begin{aligned}
& \dot{V}(t)+2 \rho V(t) \leq-2 \int_{l_{1}}^{l_{2}} \mathbf{e}_{x}^{T}(x, t) \mathbf{P}(x) \mathbf{\Theta}_{1}(x) \mathbf{e}_{x}(x, t) d x \\
& \quad-2 \int_{l_{1}}^{l_{2}} \mathbf{e}^{T}(x, t) \mathbf{P}_{x}(x) \mathbf{\Theta}_{1}(x) \mathbf{e}_{x}(x, t) d x \\
& +2 \int_{l_{1}}^{l_{2}} \mathbf{e}^{T}(x, t) \mathbf{P}(x) \mathbf{\Theta}_{2}(x) \mathbf{e}_{x}(x, t) d x \\
& +2 \int_{l_{1}}^{l_{2}} \mathbf{e}^{T}(x, t) \mathbf{P}(x) \mathbf{A}(x) \mathbf{e}(x, t) d x \\
& +\int_{l_{1}}^{l_{2}} \mathbf{e}^{T}(x, t)\left[\alpha^{-1}(x) \mathbf{P}(x) \mathbf{P}(x)+\chi \alpha(x) \mathbf{I}\right] \\
& \quad \cdot \mathbf{e}(x, t) d x+2 \rho \int_{l_{1}}^{l_{2}} \mathbf{e}^{T}(x, t) \mathbf{P}(x) \mathbf{e}(x, t) d x \\
& \quad=-\int_{l_{1}}^{l_{2}} \mathbf{e}_{x}^{T}(x, t)\left[\mathbf{P}(x) \Theta_{1}(x)+*\right] \mathbf{e}_{x}(x, t) d x \\
& +2 \int_{l_{1}}^{l_{2}} \mathbf{e}^{T}(x, t)\left[\mathbf{P}(x) \Theta_{2}(x)-\mathbf{P}_{x}(x) \Theta_{1}(x)\right]
\end{aligned}
$$




$$
\begin{aligned}
& \cdot \mathbf{e}_{x}(x, t) d x+\int_{l_{1}}^{l_{2}} \mathbf{e}^{T}(x, t)([\mathbf{P}(x) \mathbf{A}(x)+*] \\
& \left.+\alpha^{-1}(x) \mathbf{P}(x) \mathbf{P}(x)+\chi \alpha(x) \mathbf{I}+2 \rho \mathbf{P}(x)\right) \mathbf{e}(x, t) d x \\
& =\int_{l_{1}}^{l_{2}} \tilde{\mathbf{e}}^{T}(x, t) \bar{\Psi}(x) \tilde{\mathbf{e}}(x, t) d x,
\end{aligned}
$$

where $\widetilde{\mathbf{e}}(x, t) \triangleq\left[\begin{array}{ll}\mathbf{e}^{T}(x, t) & \mathbf{e}_{x}^{T}(x, t)\end{array}\right]^{T}$ and

$$
\begin{aligned}
\bar{\Psi}(x) \triangleq & {\left[\begin{array}{cc}
\bar{\Psi}_{11}(x) & \mathbf{P}(x) \boldsymbol{\Theta}_{2}(x)-\mathbf{P}_{x}(x) \boldsymbol{\Theta}_{1}(x) \\
* & -\left[\mathbf{P}(x) \mathbf{\Theta}_{1}(x)+*\right]
\end{array}\right], } \\
\bar{\Psi}_{11}(x) \triangleq & {[\mathbf{P}(x) \mathbf{A}(x)+*]+\alpha^{-1}(x) \mathbf{P}(x) \mathbf{P}(x) } \\
& +\chi \alpha(x) \mathbf{I}+2 \rho \mathbf{P}(x) .
\end{aligned}
$$

Theorem 4. Consider the unforced semilinear master-slave PDE systems (2) and (3) under Assumption 1. For a given scalar $\rho>0$, the master-slave PDE systems (2) and (3) achieve exponential synchronization with a given decay rate $\rho$, if there exist a matrix function $\mathbf{P}(x)>0$ and a scalar function $\alpha(x)>0$ satisfying the following SDLMI in space:

$$
\begin{aligned}
& \Psi(x) \\
& \triangleq\left[\begin{array}{ccc}
\Psi_{11}(x) & \mathbf{P}(x) \boldsymbol{\Theta}_{2}(x)-\mathbf{P}_{x}(x) \boldsymbol{\Theta}_{1}(x) & \mathbf{P}(x) \\
* & -\left[\mathbf{P}(x) \boldsymbol{\Theta}_{1}(x)+*\right] & 0 \\
* & * & -\alpha(x) \mathbf{I}
\end{array}\right] \\
& <0, \quad x \in\left[l_{1}, l_{2}\right]
\end{aligned}
$$

where

$$
\Psi_{11}(x)=[\mathbf{P}(x) \mathbf{A}(x)+*]+\chi \alpha(x) \mathbf{I}+2 \rho \mathbf{P}(x) .
$$

Proof. Assume that SDLMI (23) is satisfied for matrix functions $\mathbf{P}(x)>0$ and $\alpha(x)>0$. Using Schur complement, the following inequality is achieved if SDLMI (23) is satisfied for each $x \in\left[l_{1}, l_{2}\right]$ :

$$
\bar{\Psi}(x)<0, \quad x \in\left[l_{1}, l_{2}\right] .
$$

Substituting inequality (25) into (21) yields

$$
\dot{V}(t)+2 \rho V(t)<0, \quad \forall \text { non-zero } \widetilde{\mathbf{e}}(\cdot, t) .
$$

Integration of (26) from 0 to $t$ derives

$$
V(t) \leq V(0) \exp (-2 \rho t) .
$$

Since $\mathbf{P}(x)>0$ is a spatially continuous matrix function of $x$ defined on $\left[l_{1}, l_{2}\right]$, it is easily observed that $V(t)$ given by (18) satisfies the following inequality:

$$
p_{1}\|\mathbf{e}(\cdot, t)\|_{2}^{2} \leq V(t) \leq p_{2}\|\mathbf{e}(\cdot, t)\|_{2}^{2},
$$

where $p_{1} \triangleq \min _{i \in\{1,2, \ldots, n\}}\left\{\min _{x \in\left[l_{1}, l_{2}\right]}\left\{\lambda_{i}(\mathbf{P}(x))\right\}\right\}$ and $p_{2} \triangleq$ $\max _{i \in\{1,2, \ldots, n\}}\left\{\max _{x \in\left[l_{1}, l_{2}\right]}\left\{\lambda_{i}(\mathbf{P}(x))\right\}\right\}$ are two positive constants. Using (28), we can get the following relation:

$$
\begin{aligned}
p_{1}\|\mathbf{e}(\cdot, t)\|_{2}^{2} & \leq V(t) \leq V(0) \exp (-2 \rho t) \\
& \leq p_{2}\left\|\mathbf{e}_{0}(\cdot)\right\|_{2}^{2} \exp (-2 \rho t) .
\end{aligned}
$$

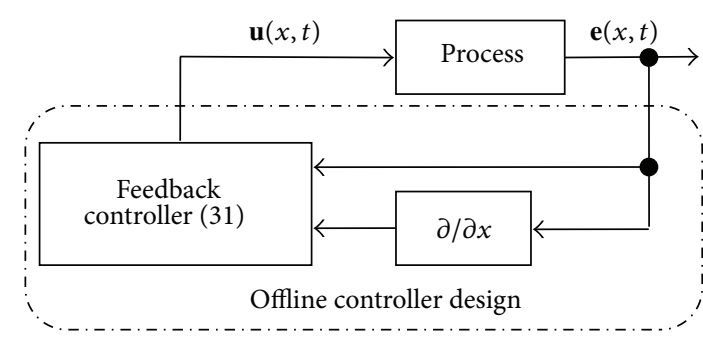

FIGURE 1: The structure of distributed P-sD state-feedback controller.

Therefore, we have

$$
\|\mathbf{e}(\cdot, t)\|_{2}^{2} \leq p_{2} p_{1}^{-1}\left\|\mathbf{e}_{0}(\cdot)\right\|_{2}^{2} \exp (-2 \rho t), \quad t \geq 0 .
$$

Thus, from (30) and Definition 2, the unforced semilinear master-slave PDE systems (2) and (3) achieve exponential synchronization with a given decay rate $\rho$. The proof is complete.

Theorem 4 presents an SDLMI-based condition for the robust exponential synchronization of the unforced semilinear master-slave PDE systems (2) and (3). Once the unforced semilinear master-slave PDE systems (2) and (3) cannot exponentially synchronize by themselves, it is desired to design a distributed P-sD controller in the semilinear masterslave PDE systems (2) and (3). Moreover, the SDLMI feasibility problem is approximately solved via the finite difference method and the existing LMI optimization techniques [41, 42].

\section{P-sD Control Design}

Once the semilinear master-slave PDE systems (2)-(3) are not exponentially synchronized by themselves, a state feedback control design is therein desired. The aim of this section is to propose a distributed $\mathrm{P}-\mathrm{sD}$ state feedback control design method to achieve exponential synchronization for semilinear master-slave PDE systems (2)-(3).

This study considers the following distributed P-sD state feedback controller $\mathcal{U}$ of the slave system $\mathcal{S}(3)$ as shown in Figure 1:

$$
\mathbf{u}(x, t)=\mathbf{K}(x) \mathbf{e}(x, t)+\mathbf{L}(x) \mathbf{e}_{x}(x, t),
$$

where $\mathbf{K}(x), \mathbf{L}(x) \in \mathfrak{R}^{m \times n}, x \in\left[l_{1}, l_{2}\right]$, are continuous matrices to be determined.

Remark 5. The controller $\mathcal{U}$ (31) of the slave system $\mathcal{S}$ needs distributed sensing and actuation, whereas it has become feasible nowadays to produce integrated control circuitry with a large number of microsensors and actuators. It has been pointed out in $[33,34,38,39]$ that the controller $\mathcal{U}$ (31) with spatial derivative term provides more spatial performance and that is why the controller $\mathcal{U}(31)$ is chosen in this paper. 
Substituting (31) into (10) gives the following closed-loop synchronization error system:

$$
\begin{aligned}
\mathbf{e}_{t}(x, t)= & \mathscr{A} \mathbf{e}(x, t)+\mathbf{A}_{c}(x) \mathbf{e}(x, t)+\mathbf{F}(\mathbf{e}(x, t)) \\
& +\mathbf{G}(x) \mathbf{L}(x) \mathbf{e}_{x}(x, t), \\
\left.\mathbf{e}_{x}(x, t)\right|_{x=l_{2}}= & \left.\mathbf{e}_{x}(x, t)\right|_{x=l_{2}}=0, \\
\mathbf{e}(x, 0)= & \mathbf{e}_{0}(x),
\end{aligned}
$$

where $\mathbf{A}_{c}(x) \triangleq \mathbf{A}(x)+\mathbf{G}(x) \mathbf{K}(x)$.

Hence, the objective of this section is to develop a design method of the distributed state feedback controller (31) to ensure exponential synchronization of the masterslave systems (2)-(3), that is, the exponential stability of the closed-loop system (32). To achieve the goal, the following assumption is necessary.

Assumption 6. The matrix $\Theta_{1}(x)$ in (2) and (3) is diagonal; that is, $\boldsymbol{\Theta}_{1}(x)=\operatorname{diag}\left\{\theta_{1,1}(x), \ldots, \theta_{1, n}(x)\right\}, x \in\left[l_{1}, l_{2}\right]$.

Assumption 6 is representative to some extent and is widely used in the literature, including the FHN equation and the tubular reactor with axial dispersion and heat exchangers $[33,34,39,44-46]$.

Consider the Lyapunov functional (16), and the time derivative of $V(t)$ along the solution of system (32) is given by

$$
\begin{aligned}
\dot{V}(t) & +2 \rho V(t) \\
= & 2 \int_{l_{1}}^{l_{2}} \mathbf{e}^{T}(x, t) \mathbf{P}(x) \mathbf{e}_{t}(x, t) d x \\
& +2 \rho \int_{l_{1}}^{l_{2}} \mathbf{e}^{T}(x, t) \mathbf{P}(x) \mathbf{e}(x, t) d x \\
= & 2 \int_{l_{1}}^{l_{2}} \mathbf{e}^{T}(x, t) \mathbf{P}(x) \mathscr{A} \mathbf{e}(x, t) d x \\
& +2 \int_{l_{1}}^{l_{2}} \mathbf{e}^{T}(x, t) \mathbf{P}(x) \mathbf{A}(x) \mathbf{e}(x, t) d x \\
& +2 \int_{l_{1}}^{l_{2}} \mathbf{e}^{T}(x, t) \mathbf{P}(x) \mathbf{F}(\mathbf{e}(x, t)) \mathbf{e}(x, t) d x \\
& +2 \int_{l_{1}}^{l_{2}} \mathbf{e}^{T}(x, t) \mathbf{P}(x) \mathbf{G}(x) \mathbf{L}(x) \mathbf{e}_{x}(x, t) d x \\
& +2 \rho \int_{l_{1}}^{l_{2}} \mathbf{e}^{T}(x, t) \mathbf{P}(x) \mathbf{e}(x, t) d x .
\end{aligned}
$$

By Assumption 1 and Lemma 3, for any positive scalar function $\beta(x) \in \mathfrak{R}, x \in\left[l_{1}, l_{2}\right]$, similar to (20), we have

$$
\begin{gathered}
2 \int_{l_{1}}^{l_{2}} \mathbf{e}^{T}(x, t) \mathbf{P}(x) \mathbf{F}(\mathbf{e}(x, t)) d x \leq \int_{l_{1}}^{l_{2}} \mathbf{e}^{T}(x, t) \\
\cdot\left[\beta(x) \mathbf{P}(x) \mathbf{P}(x)+\chi \beta^{-1}(x) \mathbf{I}\right] \mathbf{e}(x, t) d x .
\end{gathered}
$$

Substituting (19), (34) into (33) and considering $\mathbf{A}_{c}(x)$ defined in (32), we obtain

$$
\begin{aligned}
& \dot{V}(t)+2 \rho V(t) \leq-\int_{l_{1}}^{l_{2}} \mathbf{e}_{x}^{T}(x, t)\left[\mathbf{P}(x) \mathbf{\Theta}_{1}(x)+*\right] \\
& \cdot \mathbf{e}_{x}(x, t) d x+2 \int_{l_{1}}^{l_{2}} \mathbf{e}^{T}(x, t)\left[\mathbf{P}(x) \mathbf{\Theta}_{2}(x)\right. \\
& \left.-\mathbf{P}_{x}(x) \boldsymbol{\Theta}_{1}(x)+\mathbf{P}(x) \mathbf{G}(x) \mathbf{L}(x)\right] \mathbf{e}_{x}(x, t) d x \\
& +\int_{l_{1}}^{l_{2}} \mathbf{e}^{T}(x, t) \\
& \cdot([\mathbf{P}(x) \mathbf{A}(x)+\mathbf{P}(x) \mathbf{G}(x) \mathbf{K}(x)+*] \\
& \left.+\beta(x) \mathbf{P}(x) \mathbf{P}(x)+\chi \beta^{-1}(x) \mathbf{I}+2 \rho \mathbf{P}(x)\right) \mathbf{e}(x, t) d x \\
& \quad=\int_{l_{1}}^{l_{2}} \widetilde{\mathbf{e}}^{T}(x, t) \bar{\Xi}(x) \tilde{\mathbf{e}}(x, t) d x,
\end{aligned}
$$

where $\widetilde{\mathbf{e}}(x, t)$ is defined in (22) and

$$
\begin{aligned}
& \bar{\Xi}(x) \\
& \triangleq\left[\begin{array}{cc}
\bar{\Xi}_{11}(x) & \mathbf{P}(x) \mathbf{\Theta}_{2}(x)-\mathbf{P}_{x}(x) \mathbf{\Theta}_{1}(x)+\mathbf{P}(x) \mathbf{G}(x) \mathbf{L}(x) \\
* & -\left[\mathbf{P}(x) \mathbf{\Theta}_{1}(x)+*\right]
\end{array}\right], \\
& \bar{\Xi}_{11}(x) \\
& \triangleq[\mathbf{P}(x) \mathbf{A}(x)+\mathbf{P}(x) \mathbf{G}(x) \mathbf{K}(x)+*]+\beta(x) \mathbf{P}(x) \mathbf{P}(x) \\
& \quad+\chi \beta^{-1}(x) \mathbf{I}+2 \rho \mathbf{P}(x) .
\end{aligned}
$$

Theorem 7. Consider the closed-loop semilinear master-slave PDE systems (2) and (3) under Assumptions 1 and 6. For a given scalar $\rho>0$, the distributed $P$-sD controller (31) can guarantee that the master-slave PDE systems (2) and (3) achieve exponential synchronization with a given decay rate $\rho$, if there exist $n \times n$ matrix function $\mathbf{Q}(x) \triangleq \operatorname{diag}\left\{q_{1}(x), q_{2}(x), \ldots, q_{n}(x)\right\}>0, a$ scalar function $\beta(x)>0$, and $m \times n$ matrix functions $\mathbf{M}(x)$ and $\mathbf{N}(x)$ satisfying the following SDLMI in space:

$$
\begin{aligned}
& \Xi(x) \\
& \triangleq\left[\begin{array}{ccc}
\Xi_{11}(x) & \Xi_{12}(x) & \mathbf{Q}(x) \\
* & -\left[\Theta_{1}(x) \mathbf{Q}(x)+*\right] & 0 \\
* & * & -\beta(x) \chi^{-1} \mathbf{I}
\end{array}\right] \\
& \quad<0, \quad x \in\left[l_{1}, l_{2}\right],
\end{aligned}
$$

where

$$
\begin{aligned}
\Xi_{11}(x)= & {[\mathbf{A}(x) \mathbf{Q}(x)+\mathbf{G}(x) \mathbf{M}(x)+*]+\beta(x) \mathbf{I} } \\
& +2 \rho \mathbf{Q}(x), \\
\Xi_{12}(x)= & \boldsymbol{\Theta}_{2}(x) \mathbf{Q}(x)+\mathbf{G}(x) \mathbf{N}(x) \\
& -\mathbf{Q}_{x}(x) \boldsymbol{\Theta}_{1}(x) .
\end{aligned}
$$


In this case, the gain matrix functions $\mathbf{K}(x), \mathbf{L}(x), x \in\left[l_{1}, l_{2}\right]$, of the suitable controller (31) can be given as

$$
\begin{array}{r}
\mathbf{K}(x)=\mathbf{M}(x) \mathbf{Q}^{-1}(x), \\
\mathbf{L}(x)=\mathbf{N}(x) \mathbf{Q}^{-1}(x), \\
x \in\left[l_{1}, l_{2}\right] .
\end{array}
$$

Proof. Assume that SDLMI (37) is satisfied for $\mathbf{Q}(x) \triangleq$ $\operatorname{diag}\left\{q_{1}(x), q_{2}(x), \ldots, q_{n}(x)\right\}>0, \beta(x)>0, \mathbf{M}(x)$, and $\mathbf{N}(x)$. Using the Schur complement, SDLMI (37) is equivalent to the inequality

$$
\widetilde{\Xi}(x) \triangleq\left[\begin{array}{cc}
\widetilde{\Xi}_{11}(x) & \Xi_{12}(x) \\
* & -\left[\boldsymbol{\Theta}_{1}(x) \mathbf{Q}(x)+*\right]
\end{array}\right]<0,
$$

where $\widetilde{\Xi}_{11}(x)=[\mathbf{A}(x) \mathbf{Q}(x)+\mathbf{G}(x) \mathbf{M}(x)+*]+\beta(x) \mathbf{I}+$ $\chi \beta^{-1}(x) \mathbf{Q}(x) \mathbf{Q}(x)+2 \rho \mathbf{Q}(x)$.

Letting

$$
\begin{aligned}
\mathbf{P}(x) & =\mathbf{Q}^{-1}(x) \\
\mathbf{M}(x) & =\mathbf{K}(x) \mathbf{Q}(x), \\
\mathbf{N}(x) & =\mathbf{L}(x) \mathbf{Q}(x),
\end{aligned}
$$

pre- and postmultiplying (40) by $\operatorname{diag}\left\{\mathbf{Q}^{-1}(x), \mathbf{Q}^{-1}(x)\right\}$, respectively, with application of Assumption 6, and considering the property $\boldsymbol{\Theta}_{1}(x) \mathbf{Q}(x)=\mathbf{Q}(x) \Theta_{1}(x), \mathbf{Q}_{x}(x)=$ $-\mathbf{Q}(x) \mathbf{Q}_{x}^{-1}(x) \mathbf{Q}(x)$, and the commutative law of matrix multiplication [47], we can obtain

$$
\begin{aligned}
\bar{\Xi}(x)= & \operatorname{diag}\left\{\mathbf{Q}^{-1}(x), \mathbf{Q}^{-1}(x)\right\} \\
\cdot \widetilde{\Xi}(x) \operatorname{diag}\left\{\mathbf{Q}^{-1}(x), \mathbf{Q}^{-1}(x)\right\}, & \\
& x \in\left[l_{1}, l_{2}\right] .
\end{aligned}
$$

Since $\operatorname{diag}\left\{\mathbf{Q}^{-1}(x), \mathbf{Q}^{-1}(x)\right\}>0, x \in\left[l_{1}, l_{2}\right]$, we can get the following inequality from (40) and (42):

$$
\bar{\Xi}(x)<0, \quad x \in\left[l_{1}, l_{2}\right] .
$$

Substituting inequality (43) into (35) yields

$$
\dot{V}(t)+2 \rho V(t)<0, \quad \forall \text { non-zero } \widetilde{\mathbf{e}}(\cdot, t) .
$$

The rest of proof of Theorem 7 is similar to that of Theorem 4, and thus we can also get the conclusion that the semilinear master-slave PDE systems (2) and (3) achieve exponential synchronization with a given decay rate $\rho$ with the suitable controller (31). Moreover, from (41), we have (39). The proof is complete.

Theorem 7 presents an SDLMI-based condition for the existence of a distributed controller (31) for the exponential synchronization of the semilinear master-slave PDE systems (2) and (3). Explicit expressions of a desired controller are proposed when the SDLMI (37) is feasible.

Remark 8. Notice that an SDLMI control design has been more recently proposed in $[33,34]$ for a class of PDE systems.
Different from the stable control design for PDE system in $[33,34]$, this paper provides an SDLMI-based sufficient condition on the exponential synchronization for masterslave PDE systems.

Remark 9. Notice that [38-40] dealt with exponential synchronization of linear models, while this paper considers exponential synchronization of the model with nonlinear perturbation.

Remark 10. For a special case, when the master system $\mathscr{M}$ : $\mathbf{y}_{t}(x, t) \equiv 0$, we have $\mathbf{z}(x, t)=\mathbf{e}(x, t)$, and then the exponential synchronization of the master-slave systems is equal to exponential stability of the slave system $\mathcal{S}$. In other words, Theorem 4 provides the sufficient condition of exponential stability of the slave system $\delta$ with a given decay rate $\rho$ when the master system $\mathrm{y}(x, t) \equiv 0$, while Theorem 7 also contains the way of the P-sD controller (31) design for exponential stability.

\section{Numerical Simulation}

In this section, in order to show the effectiveness of Theorems 4 and 7, we consider the following two examples.

Example 1. Consider semilinear master-slave PDE systems (2) and (3) with the following spatial variable coefficients:

$$
\begin{aligned}
& \mathbf{y}(x, t)=\left[y_{1}(x, t), y_{2}(x, t)\right]^{T}, \\
& \mathbf{z}(x, t)=\left[z_{1}(x, t), z_{2}(x, t)\right]^{T}, \\
& \boldsymbol{\Theta}_{1}(x)=\left[\begin{array}{ll}
1 & 0 \\
0 & 4
\end{array}\right] \text {, } \\
& \boldsymbol{\Theta}_{2}(x)=\left[\begin{array}{ll}
1 & 0 \\
0 & 1
\end{array}\right] \text {, } \\
& \mathbf{A}(x)=\left[\begin{array}{cc}
-2.7-\exp \left(-\frac{x}{L}\right) & 0.5 \\
-2 \cos (x) & -3.7
\end{array}\right] \text {, } \\
& l_{1}=0 \text {, } \\
& l_{2}=10 \text {, } \\
& \mathbf{f}(\mathbf{y}(x, t), x, t)=\left[\begin{array}{c}
-0.25 y_{1}^{5} \\
0
\end{array}\right] \text {, } \\
& \mathbf{f}(\mathbf{z}(x, t), x, t)=\left[\begin{array}{c}
-0.25 z_{1}^{5} \\
0
\end{array}\right],
\end{aligned}
$$

and the following initial conditions:

$$
\begin{aligned}
& \mathbf{y}_{0}(x)=\left[\begin{array}{c}
-0.2+0.6 \cos \left(\frac{\pi x}{\mathrm{~L}}\right) \\
-0.7 \sin \left(\frac{\pi x}{\mathrm{~L}}+\frac{\pi}{2}\right)
\end{array}\right], \\
& \mathbf{z}_{0}(x)=\left[\begin{array}{c}
0.5 \cos \left(\frac{2 \pi x}{L}\right) \\
-0.3+0.1 \cos \left(\frac{\pi x}{L}\right)
\end{array}\right],
\end{aligned}
$$

where $L \triangleq l_{2}-l_{1}=10$. 

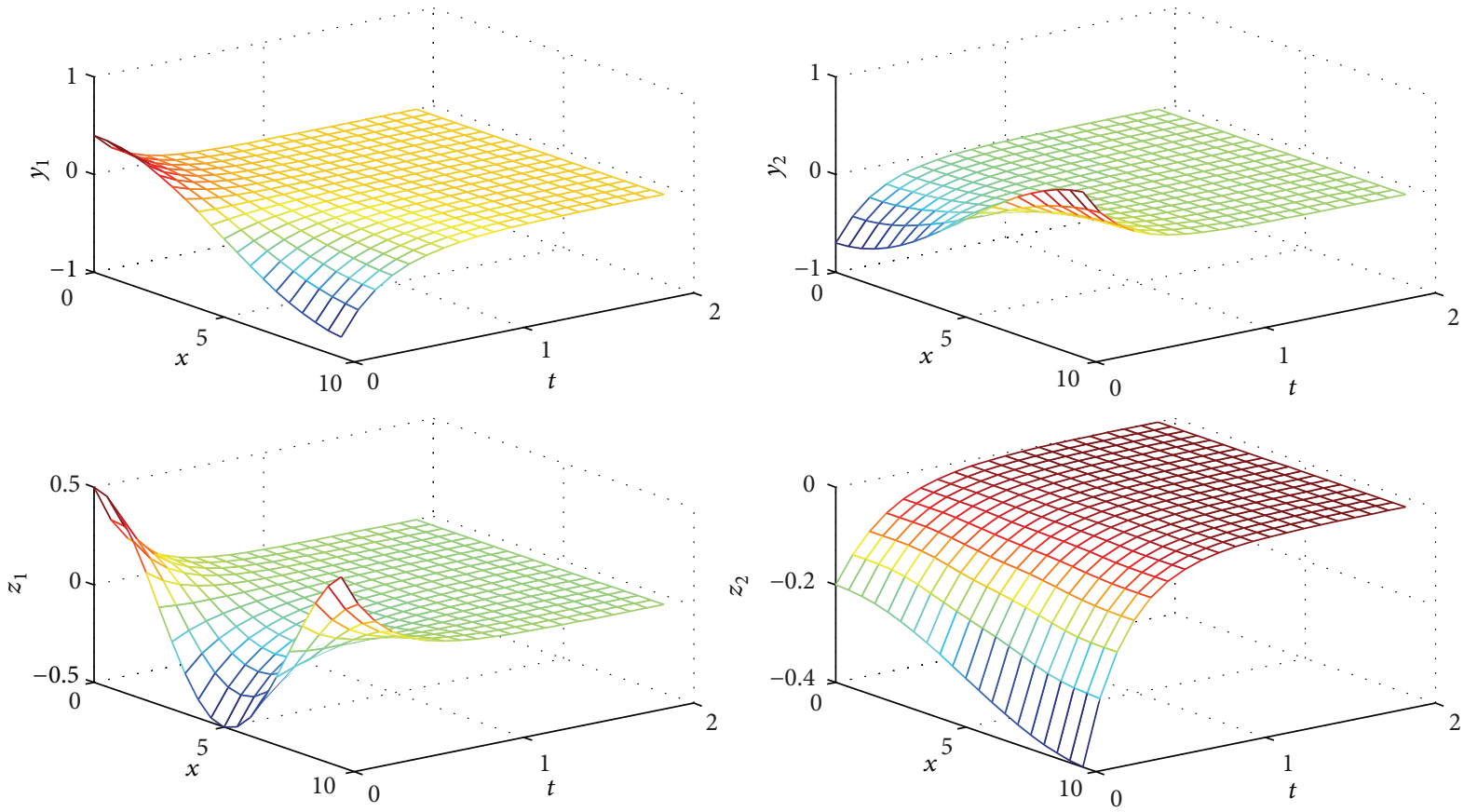

FIgURE 2: The unforced profile of evolution of the master-slave PDE systems (2) and (3).

Let $\Omega \triangleq\left\{\widetilde{\mathbf{y}}(x, t) \mid-0.8 \leq \tilde{y}_{i}(x, t) \leq 0.8, \tilde{y}_{i}(x, t) \in\right.$ $\left.\left\{y_{i}(x, t), z_{i}(x, t)\right\}, x \in[0,10], t \geq 0, i=1,2\right\}$. The parameter $\chi$ satisfying Assumption 1 is chosen as

$$
\begin{aligned}
\chi \triangleq & \max _{\mathbf{y}(x, t), \mathbf{z}(x, t) \in \Omega}\left\{\left\|\frac{\partial \mathbf{f}(\mathbf{y}(x, t), x, t)}{\partial \mathbf{y}(x, t)}\right\|^{2},\right. \\
& \left.\left\|\frac{\partial \mathbf{f}(\mathbf{z}(x, t), x, t)}{\partial \mathbf{z}(x, t)}\right\|^{2}\right\}=0.0625 \\
& \max _{\mathbf{y}(x, t), \mathbf{z}(x, t) \in \Omega}\left\{25 y_{1}^{4}(x, t), 25 z_{1}^{4}(x, t)\right\}=6.5536 .
\end{aligned}
$$

Divide the spatial domain $[0,10]$ into space instances $\left\{x_{k}, k \in\{0,1,2, \ldots, 100\}, x_{0}=0, x_{100}=10\right\}$ of the same distance, where $x_{k}-x_{k-1}=0.1$. With application of Theorem 4, employing Algorithm 1 in [34] to solve SDLMI (23) and setting $\mathbf{Q}\left(x_{-1}\right)=\mathbf{I}$ and $\rho=0.1$, we get feasible solutions of $\mathbf{P}(x)>0$ and $\alpha(x)>0$. Therefore, the unforced semilinear master-slave PDE systems (2) and (3) with coefficients (45) and initial conditions (46) achieve robust exponential synchronization with a given decay rate $\rho$, which is apparent from Figure 2.

Example 2. Consider semilinear master-slave PDE systems (2) and (3) with the following spatially variable coefficients:

$$
\begin{aligned}
& \mathbf{y}(x, t)=\left[y_{1}(x, t), y_{2}(x, t)\right]^{T}, \\
& \mathbf{z}(x, t)=\left[z_{1}(x, t), z_{2}(x, t)\right]^{T}, \\
& \mathbf{\Theta}_{1}(x)=\left[\begin{array}{ll}
1 & 0 \\
0 & 4
\end{array}\right],
\end{aligned}
$$

$$
\begin{aligned}
\boldsymbol{\Theta}_{2}(x) & =\left[\begin{array}{ll}
0 & 0 \\
0 & 0
\end{array}\right], \\
\mathbf{A}(x) & =\left[\begin{array}{cc}
1 & \left.2 \exp \left(-\frac{x}{L}\right)\right], \\
\sin (x) & -3
\end{array}\right] \\
l_{1} & =0, \\
l_{2} & =20, \\
\mathbf{G}(x) & =\left[\begin{array}{c}
1 \\
0.5
\end{array}\right], \\
\mathbf{f}(\mathbf{y}(x, t), x, t) & =\left[\begin{array}{c}
-0.5 y_{1}^{3} \\
0
\end{array}\right], \\
\mathbf{f}(\mathbf{z}(x, t), x, t) & =\left[\begin{array}{c}
-0.5 z_{1}^{3} \\
0
\end{array}\right],
\end{aligned}
$$

and the following initial conditions:

$$
\begin{aligned}
& \mathbf{y}_{0}(x)=\left[\begin{array}{c}
\left.0.6 \cos \left(\frac{\pi x}{L}\right)\right] \\
-0.4
\end{array}\right] \\
& \mathbf{z}_{0}(x)=\left[\begin{array}{c}
\left.0.5 \cos \left(\frac{2 \pi x}{L}\right)\right] \\
-0.2
\end{array}\right]
\end{aligned}
$$

where $L \triangleq l_{2}-l_{1}=20$.

Figure 3 shows the unforced profiles of evolution of the semilinear master-slave PDE systems (2) and (3) with the parameters given in (40) under the initial condition given in (41). It is apparent from Figure 3 that the operating steady 

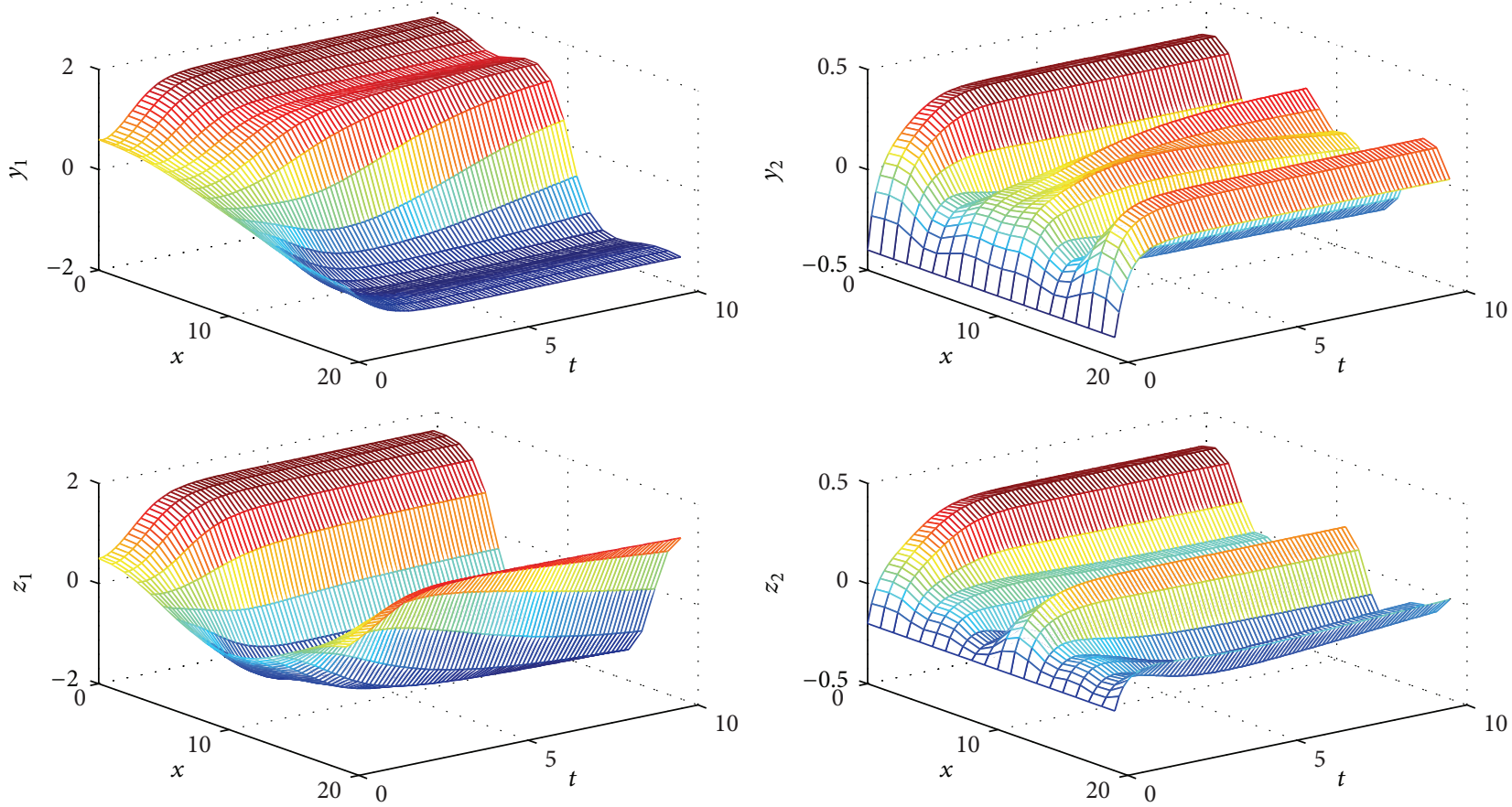

FIgURE 3: The unforced profile of evolution of the master-slave PDE systems (2) and (3).

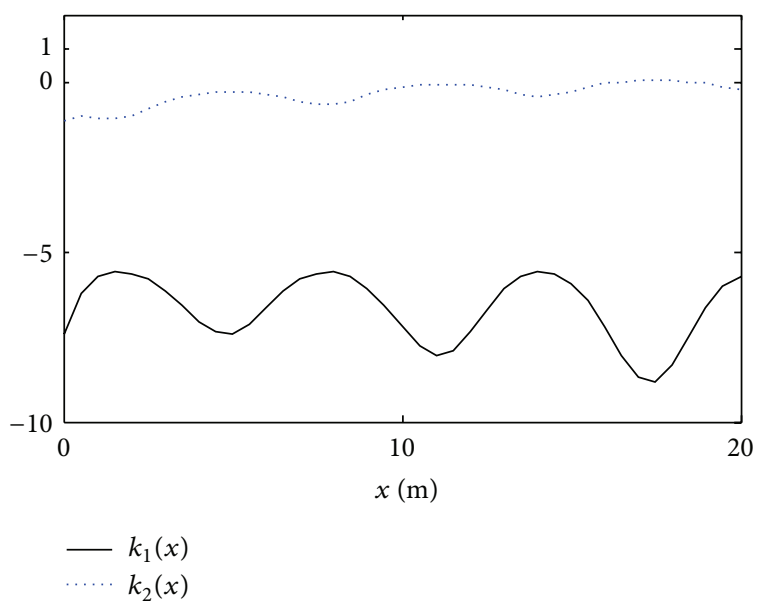

Figure 4: Approximated control gain matrix $\mathbf{K}(x)$.

state $\mathbf{y}(x, t)$ of the master PDE system (2) and the operating steady state $\mathbf{z}(x, t)$ of the slave PDE system (3) are not synchronized.

Let $\Omega \triangleq\left\{\widetilde{\mathbf{y}}(x, t) \mid-1.7 \leq \tilde{y}_{i}(x, t) \leq 1.7, \tilde{y}_{i}(x, t) \in\right.$ $\left.\left\{y_{i}(x, t), z_{i}(x, t)\right\}, x \in[0,20], t \geq 0, i=1,2\right\}$. The parameter $\chi$ satisfying Assumption 1 is chosen as

$$
\begin{aligned}
\chi \triangleq & \max _{\mathbf{y}(x, t), \mathbf{z}(x, t) \in \Omega}\left\{\left\|\frac{\partial \mathbf{f}(\mathbf{y}(x, t), x, t)}{\partial \mathbf{y}(x, t)}\right\|^{2},\right. \\
& \left.\left\|\frac{\partial \mathbf{f}(\mathbf{z}(x, t), x, t)}{\partial \mathbf{z}(x, t)}\right\|^{2}\right\}=0.09 \\
& \cdot \max _{\mathbf{y}(x, t), \mathbf{z}(x, t) \in \Omega}\left\{9 y_{1}^{4}(x, t), 9 z_{1}^{4}(x, t)\right\}=6.5025 .
\end{aligned}
$$

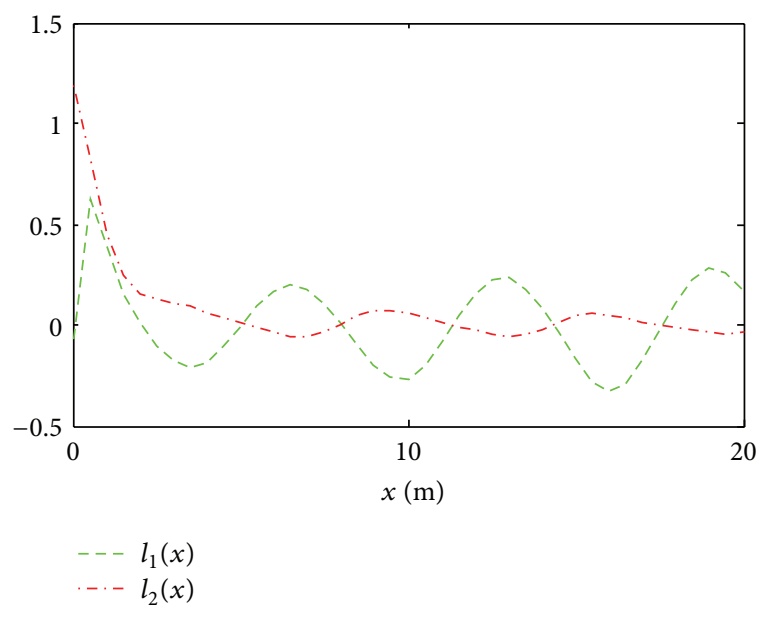

FIGURE 5: Approximated control gain matrix $\mathbf{L}(x)$.

In this example, the distributed $\mathrm{P}$-sD state feedback controller (31) is employed, where $\mathbf{K}(x) \triangleq\left[k_{1}(x) k_{2}(x)\right]$, $\mathbf{L}(x) \triangleq\left[l_{1}(x) l_{2}(x)\right], x \in[0,20]$, are $1 \times 2$ matrix functions to be determined. Divide the spatial domain $[0,20]$ into space instances $\left\{x_{k}, k \in\{0,1,2, \ldots, 40\}, x_{0}=0, x_{40}=20\right\}$ of the same distance, where $x_{k}-x_{k-1}=0.5$. With application to Theorem 7, employing Algorithm 1 in [34] to solve SDLMI (37) and setting $\mathbf{Q}\left(x_{-1}\right)=0.3 \mathbf{I}$ and $\rho=0.1$, we get feasible solutions of $\mathbf{Q}(x) \triangleq \operatorname{diag}\left\{q_{1}(x), q_{2}(x), \ldots, q_{n}(x)\right\}>0, \beta(x)>$ $0, \mathbf{M}(x)$, and $\mathbf{N}(x)$. Thereby, we can get the approximated control gain matrix functions $\mathbf{K}(x)$ and $\mathbf{L}(x)$ by (39) as shown in Figures 4 and 5, respectively.

Applying the controller (31) with the approximated control gain matrices $\mathbf{K}(x)$ and $\mathbf{L}(x)$ shown in Figures 4 and 5 

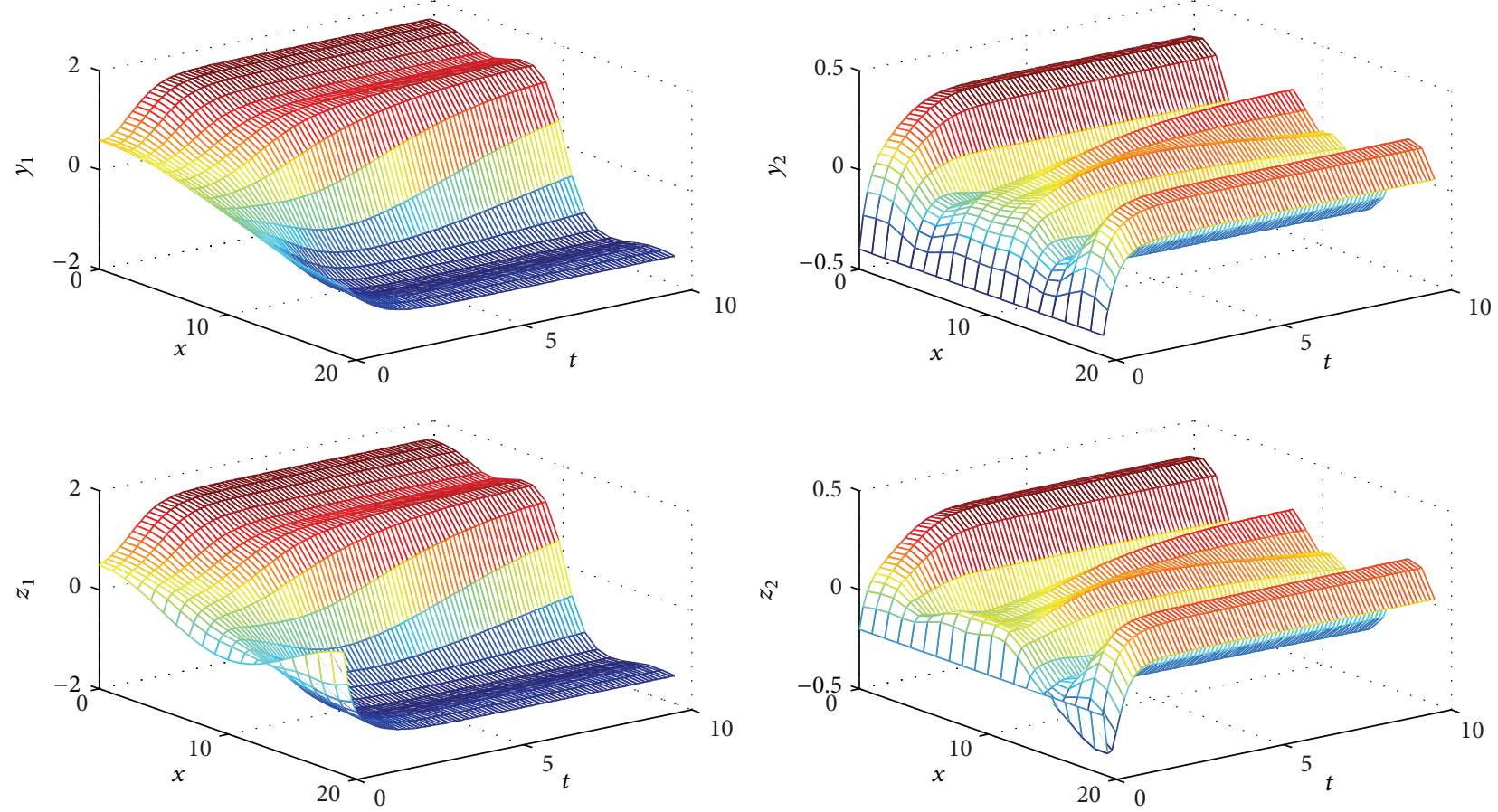

FIGURE 6: Close-loop profile of evolution of the master-slave PDE systems (2) and (3).

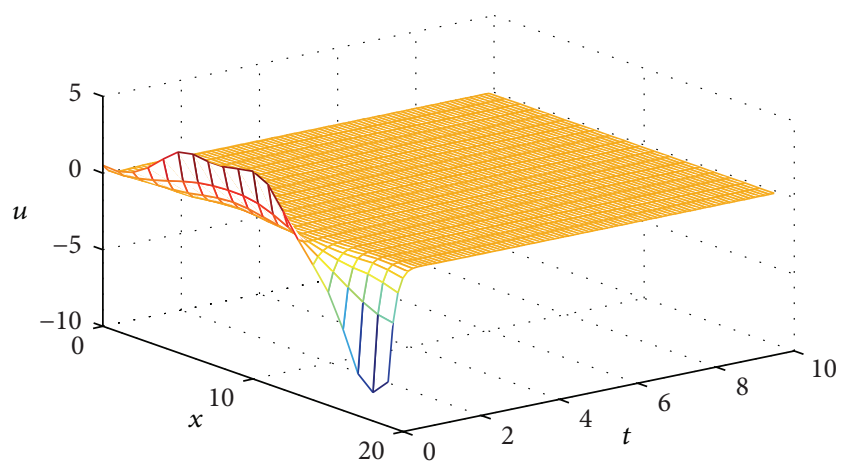

FIGURE 7: Evolution profile of the distributed control $u(x, t)$.

to the semilinear master-slave PDE systems (2) and (3) with the parameters given in (48) under the initial condition given in (49), Figure 6 indicates the closed-loop profiles of evolution of the semilinear master-slave PDE systems of $\mathbf{y}(x, t)=\left[y_{1}(x, t), y_{2}(x, t)\right]^{T}$ and $\mathbf{z}(x, t)=\left[z_{1}(x, t), z_{2}(x, t)\right]^{T}$, respectively.

It is clear from Figure 6 that the proposed distributed state feedback controller (31) can ensure that the semilinear master-slave PDE systems (2) and (3) with the parameters given in (48) under the initial condition given in (49) achieve exponential synchronization with a given decay rate $\rho$. Moreover, the evolution profile of the distributed control $\mathbf{u}(x, t)$ is shown in Figure 7.

\section{Conclusions}

In this paper, we have addressed the exponential synchronization problem of semilinear master-slave PDE systems with spatially variable coefficients. An SDLMI-based sufficient condition for the robust exponential synchronization of the unforced semilinear master-slave PDE systems has been presented. Moreover, when the master-slave PDE systems are not exponentially synchronized, it has been proved in detail that P-sD controllers with appropriate gain parameters can guide the closed-loop master-slave PDE systems to exponentially synchronize with a given decay rate $\rho$ if a given SDLMI condition is fulfilled. Finally, numerical examples showed the effectiveness of the proposed methods.

\section{Conflict of Interests}

The authors declare that there is no conflict of interests regarding the publication of this paper.

\section{Acknowledgments}

This work was supported in part by the National Natural Science Foundation of China under Grant nos. 61273012, 61403179, and 61304023, in part by the Natural Science Foundation of Shandong Province of China under Grant nos. ZR2014AL009 and ZR2014CP008, in part by the Science and Technology Development Plan Project of Shandong Province under Grant no. 2012YD01052, and in part by the Applied Mathematics Enhancement Program of Linyi University. 


\section{References}

[1] W. Wu, D. Liu, J. Liu, and J. Wu, "Master-slave intelligent robot telepresence system," Computers and Industrial Engineering, vol. 31, no. 3-4, pp. 703-706, 1996.

[2] N.-V.-Q. Hung, T. Narikiyo, and H.-D. Tuan, "Nonlinear adaptive control of master-slave system in teleoperation," Control Engineering Practice, vol. 11, no. 1, pp. 1-10, 2003.

[3] A. Ghorbanian, S.-M. Rezaei, A.-R. Khoogar, M. Zareinejad, and K. Baghestan, "A novel control framework for nonlinear time-delayed Dual-master/Single- slave teleoperation," ISA Transactions, vol. 52, no. 2, pp. 268-277, 2013.

[4] K. Kosuge and J. Ishikawa, "Task-oriented control of singlemaster multi-slave manipulator system," Robotics and Autonomous Systems, vol. 12, no. 1-2, pp. 95-105, 1994.

[5] R. Tadakuma, Y. Asahara, H. Kajimoto, N. Kawakami, and S. Tachi, "Development of anthropomorphic multi-D.O.F. masterslave arm for mutual telexistence," IEEE Transactions on Visualization and Computer Graphics, vol. 11, no. 6, pp. 626-636, 2005.

[6] D. French, W.-W. Lu, and Z.-X. Yang, "A master-slave controller for a flexible manufacturing system," CIRP AnnalsManufacturing Technology, vol. 31, no. 1, pp. 397-400, 1982.

[7] A. Jafari, H. Sedghi, K. Mabhouti, and S. Behnia, "Slave-master dynamics of semiconductor laser with short external cavity," Optics Communications, vol. 284, no. 12, pp. 3018-3029, 2011.

[8] C.-S. Chang, H.-B. Quek, and J.-B. X. Devotta, "Power system excitation control using master-slave fuzzy power system stabilisers," Fuzzy Sets and Systems, vol. 102, no. 1, pp. 85-94, 1999.

[9] M. Depolli, R. Trobec, and B. Filipič, "Asynchronous masterslave parallelization of differential evolution for multi-objective optimization," Evolutionary Computation, vol. 21, no. 2, pp. 261291, 2013.

[10] S. Strogatz, Sync: The Emerging Science of Spontaneous Order, Hyperion, New York, NY, USA, 2003.

[11] X. Jian, Y. Yang, and J. Long, "Synchronisation of complex networks with derivative coupling via adaptive control," International Journal of Systems Science. Principles and Applications of Systems and Integration, vol. 44, no. 12, pp. 2183-2189, 2013.

[12] M. Liu, S. Zhang, Z. Fan, S. Zheng, and W. Sheng, "Exponential $\mathrm{H}_{\infty}$ synchronization and state estimation for chaotic systems via a unified model," IEEE Transactions on Neural Networks and Learning Systems, vol. 24, no. 7, pp. 1114-1126, 2013.

[13] W. He, F. Qian, Q.-L. Han, and J. Cao, "Synchronization error estimation and controller design for delayed Lur'e systems with parameter mismatches," IEEE Transactions on Neural Networks and Learning Systems, vol. 23, no. 10, pp. 1551-1563, 2012.

[14] L.-M. Pecora and T.-L. Carroll, "Synchronization in chaotic systems," Physical Review Letters, vol. 64, no. 8, pp. 821-824, 1990.

[15] H.-K. Chen, "Global chaos synchronization of new chaotic systems via nonlinear control," Chaos, Solitons and Fractals, vol. 23, no. 4, pp. 1245-1251, 2005.

[16] Q.-L. Han, "On designing time-varying delay feedback controllers for master-slave synchronization of Lur'e systems," IEEE Transactions on Circuits and Systems. I. Regular Papers, vol. 54, no. 7, pp. 1573-1583, 2007.

[17] H. Guo, S. Zhong, and F. Gao, "Design of PD controller for master-slave synchronization of Lur'e systems with time-delay," Applied Mathematics and Computation, vol. 212, no. 1, pp. 8693, 2009.
[18] Y.-J. Wang, J.-N. Hao, and Z.-Q. Zuo, "Exponential synchronization of master-slave lure systems via intermittent Timedelay feedback control," Communications in Theoretical Physics, vol. 54, no. 4, pp. 679-686, 2010.

[19] W.-H. Chen, Z. Wang, and X. Lu, "On sampled-data control for master-slave synchronization of chaotic Lur'e systems," IEEE Transactions on Circuits and Systems II: Express Briefs, vol. 59, no. 8, pp. 515-519, 2012.

[20] H. Mkaouar and O. Boubaker, "Chaos synchronization for master slave piecewise linear systems: application to Chua's circuit," Communications in Nonlinear Science and Numerical Simulation, vol. 17, no. 3, pp. 1292-1302, 2012.

[21] H.-H. Kuo, T.-L. Liao, and J.-J. Yan, "Design and synchronization of master-slave electronic horizontal platform system," Discrete Dynamics in Nature and Society, vol. 2012, Article ID 948126, 11 pages, 2012.

[22] K. Ding and Q.-L. Han, "Master-slave synchronization criteria for horizontal platform systems using time delay feedback control," Journal of Sound and Vibration, vol. 330, no. 11, pp. 2419-2436, 2011.

[23] H.-R. Karimi and H. Gao, "LMI-based $H_{\infty}$ synchronization of second-order neutral master-slave systems using delayed output feedback control," International Journal of Control, Automation and Systems, vol. 7, no. 3, pp. 371-380, 2009.

[24] C.-W. Wu, C.-F. Hsu, and C.-K. Hwang, "Master-slave chaos synchronization using adaptive TSK-type CMAC neural control," Journal of the Franklin Institute. Engineering and Applied Mathematics, vol. 348, no. 8, pp. 1847-1868, 2011.

[25] Y. Ge, T. Li, and S. Fei, "Master-slave synchronization of stochastic neural networks with mixed time-varying delays," Mathematical Problems in Engineering, vol. 2012, Article ID 730941, 18 pages, 2012.

[26] A. Loría, "Master-slave synchronization of fourth-order lü chaotic oscillators via linear output feedback," IEEE Transactions on Circuits and Systems II: Express Briefs, vol. 57, no. 3, pp. 213-217, 2010.

[27] S. Oancea, F. Grosu, A. Lazar, and I. Grosu, "Master-slave synchronization of Lorenz systems using a single controller," Chaos, Solitons and Fractals, vol. 41, no. 5, pp. 2575-2580, 2009.

[28] G. Feng and J. Cao, "Master-slave synchronization of chaotic systems with a modified impulsive controller," Advances in Difference Equations, vol. 2013, article 24, 2013.

[29] P. García, A. Acosta, and H. Leiva, "Synchronization conditions for master-slave reaction diffusion systems," Europhysics Letters, vol. 88, no. 6, Article ID 60006, 4 pages, 2009.

[30] J.-W. Wang, H.-N. Wu, and H.-X. Li, "Distributed fuzzy control design of nonlinear hyperbolic pde systems with application to nonisothermal plug-flow reactor," IEEE Transactions on Fuzzy Systems, vol. 19, no. 3, pp. 514-526, 2011.

[31] H.-N. Wu, J.-W. Wang, and H.-X. Li, "Design of distributed $H_{\infty}$ fuzzy controllers with constraint for nonlinear hyperbolic PDE systems," Automatica, vol. 48, no. 10, pp. 2535-2543, 2012.

[32] H.-N. Wu, J.-W. Wang, and H.-X. Li, "Exponential stabilization for a class of nonlinear parabolic PDE systems via fuzzy control approach," IEEE Transactions on Fuzzy Systems, vol. 20, no. 2, pp. 318-329, 2012.

[33] C.-D. Yang, J. Qiu, and J.-W. Wang, "Robust $H_{\infty}$ control for a class of nonlinear distributed parameter systems via proportional-spatial derivative control approach," Abstract and Applied Analysis, vol. 2014, Article ID 631071, 8 pages, 2014. 
[34] J.-W. Wang, H.-N. Wu, and H.-X. Li, "Distributed proportionalspatial derivative control of nonlinear parabolic systems via fuzzy PDE modeling approach," IEEE Transactions on Systems, Man, and Cybernetics, Part B: Cybernetics, vol. 42, no. 3, pp. 927938, 2012.

[35] L. Sheng and M. Gao, "Synchronization of delayed BAM neural networks with reaction-diffusion terms," in Proceedings of the 30th Chinese Control Conference, pp. 2753-2758, Yantai, China, July 2011.

[36] L. Sheng, H. Yang, and X. Lou, "Adaptive exponential synchronization of delayed neural networks with reaction-diffusion terms," Chaos, Solitons \& Fractals, vol. 40, no. 2, pp. 930-939, 2009.

[37] K. Wang, Z. Teng, and H. Jiang, "Global exponential synchronization in delayed reaction-diffusion cellular neural networks with the Dirichlet boundary conditions," Mathematical and Computer Modelling, vol. 52, no. 1-2, pp. 12-24, 2010.

[38] K. Yuan, A. Alofi, J. Cao, A. Al-Mazrooei, and A. Elaiw, "Synchronization of the coupled distributed parameter system with time delay via proportional-spatial derivative control," Discrete Dynamics in Nature and Society, vol. 2014, Article ID 418258, 7 pages, 2014.

[39] C.-D. Yang, J.-L. Qiu, and H.-B. He, "Exponential synchronization for a class of complex spatio-temporal networks with space-varying coefficients," Neurocomputing, vol. 151, pp. 401407, 2015.

[40] J.-W. Wang, C.-D. Yang, and C.-Y. Sun, "Exponential synchronization for a class of networked linear parabolic PDE systems via boundary control," in Proceedings of the International Joint Conference on Neural Networks, pp. 3981-3986, Beijing, China, 2014.

[41] S. Boyd, L. El Ghaoui, E. Feron, and V. Balakrishnan, Linear Matrix Inequalities in System and Control Theory, SIAM, Philadelphia, Pa, USA, 1994.

[42] P. Gahinet, A. Nemirovskii, A.-J. Laub, and M. Chilali, LMI Control Toolbox for Use with Matlab, The MathWorks, Natick, Mass, USA, 1995.

[43] A. Pazy, Semigroups of Linear Operators and Applications to Partial Differential Equations, Springer, New York, NY, USA, 1983.

[44] J. Keener and J. Sneyd, Mathematical Physiology, vol. 8 of Interdisciplinary Applied Mathematics, Springer, New York, NY, USA, 1998.

[45] Y. Orlov and D. Dochain, "Discontinuous feedback stabilization of minimum-phase semilinear infinite-dimensional systems with application to chemical tubular reactor," IEEE Transactions on Automatic Control, vol. 47, no. 8, pp. 1293-1304, 2002.

[46] C. Theodoropoulos, Y.-H. Qian, and I.-G. Kevrekidis, "'Coarse’ stability and bifurcation analysis using time-steppers: a reaction-diffusion example," Proceedings of the National Academy of Sciences of the United States of America, vol. 97, no. 18, pp. 98409843, 2000.

[47] K.-M. Abadir and J.-R. Magnus, Matrix Algebra, vol. 1 of Econometric Exercises, Cambridge University Press, Cambridge, UK, 2005. 


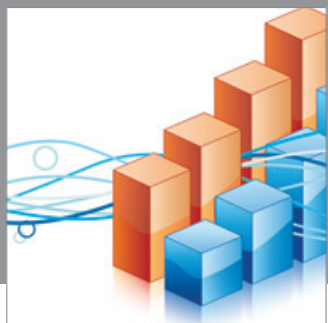

Advances in

Operations Research

mansans

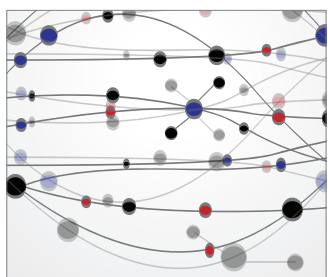

The Scientific World Journal
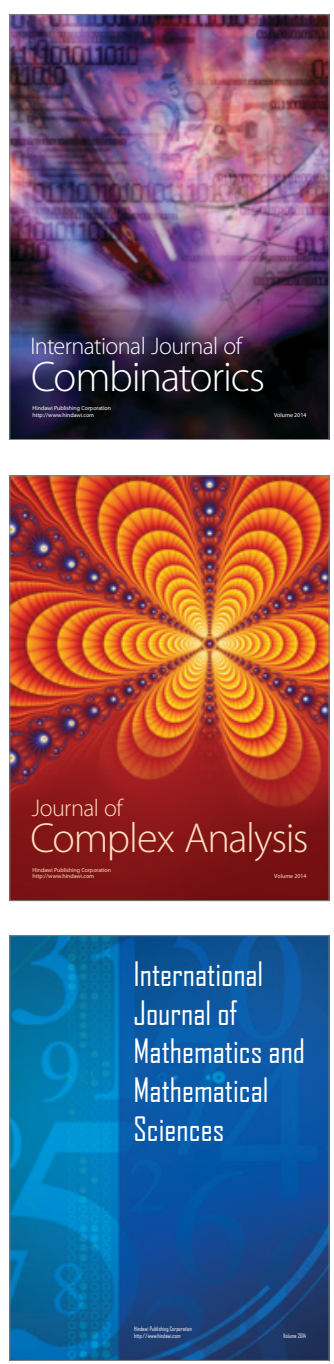
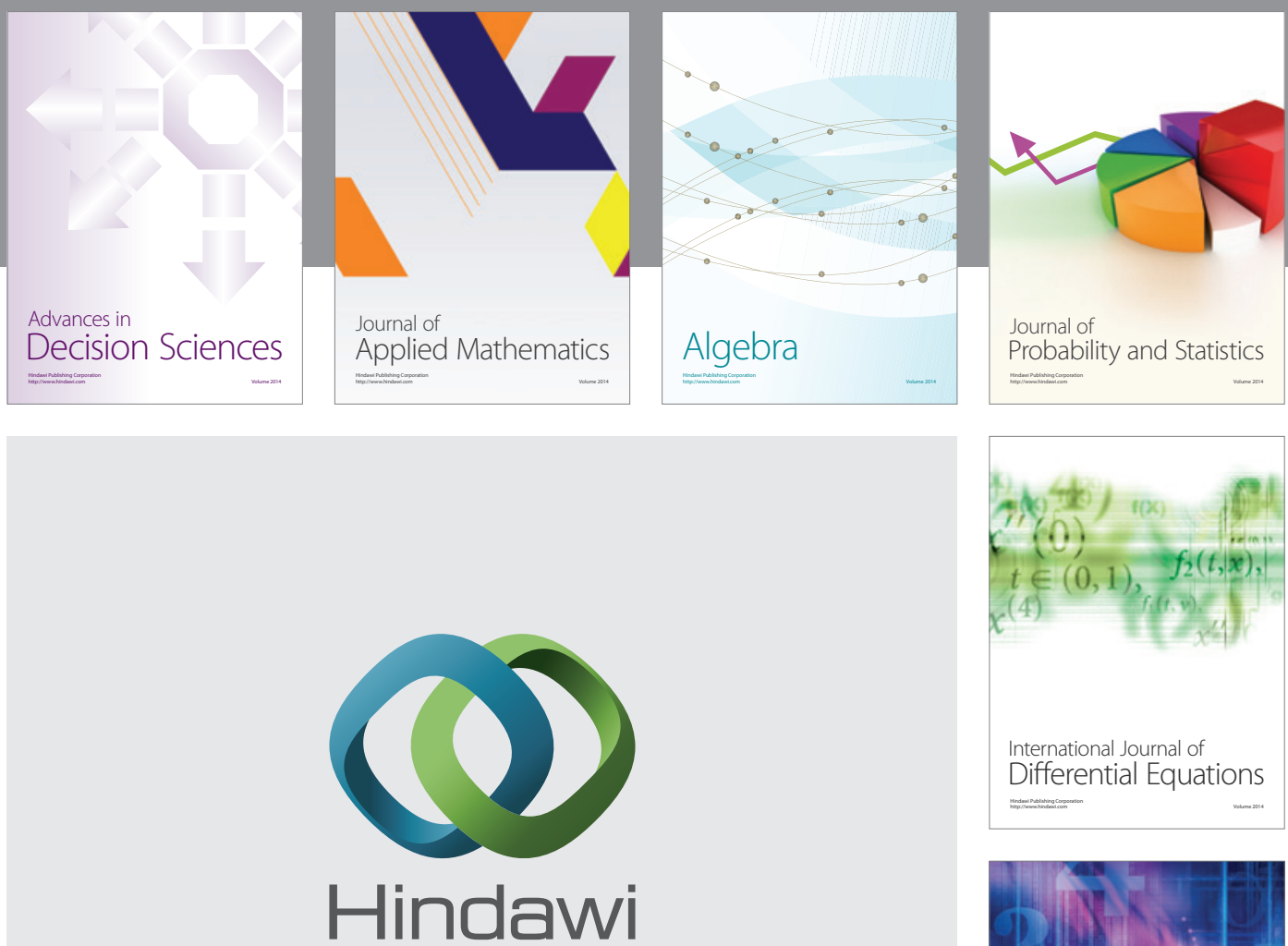

Submit your manuscripts at http://www.hindawi.com
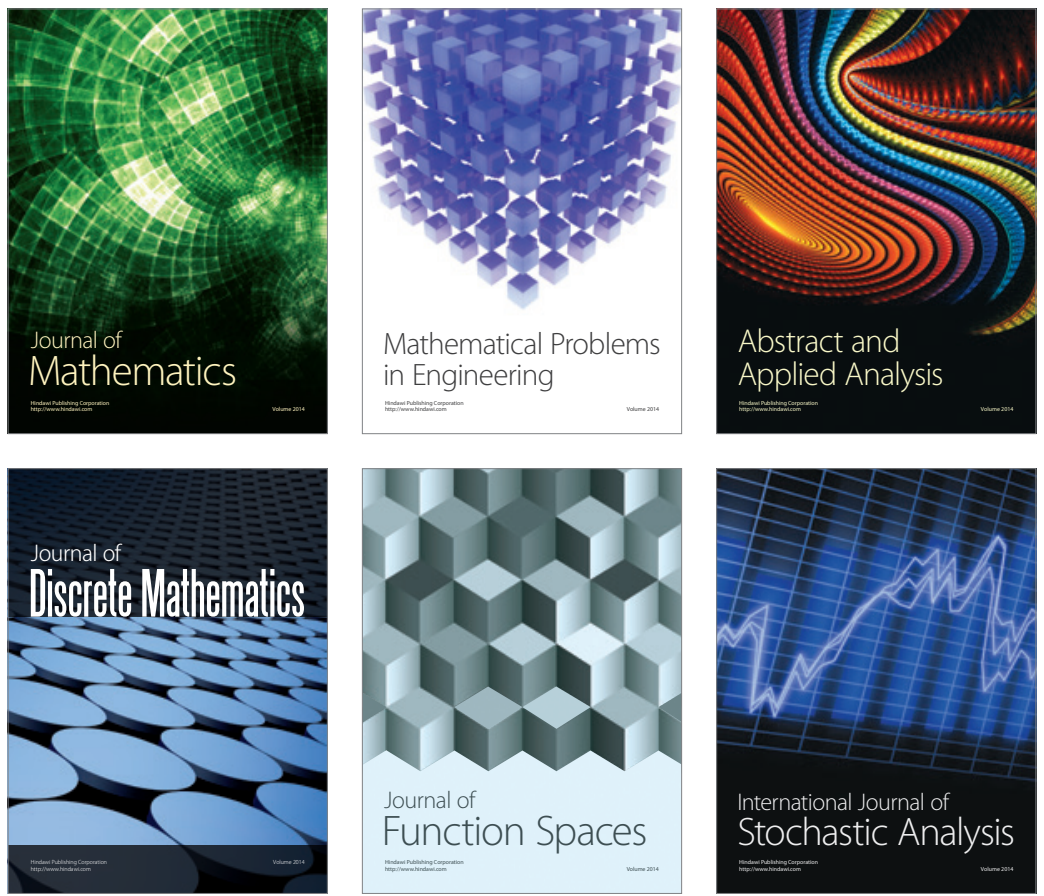

Journal of

Function Spaces

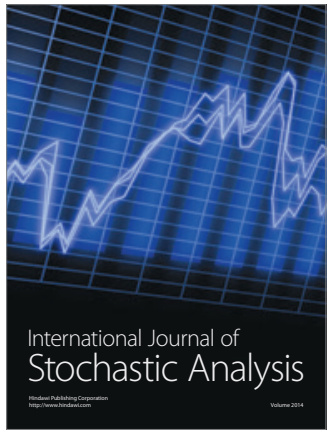

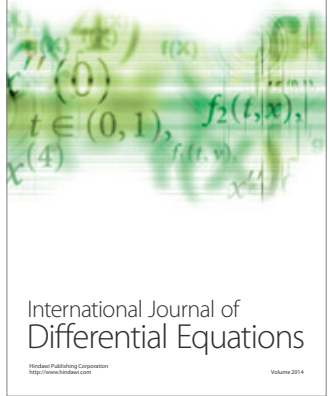
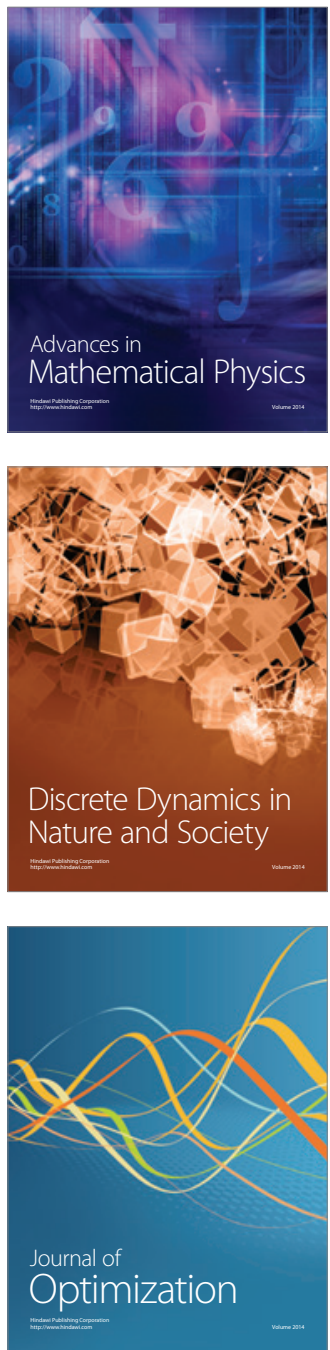